\title{
Age differences in components of mental-rotation task performance
}

\author{
CHRISTOPHER HERTZOG and BART RYPMA \\ Georgia Institute of Technology, Atlanta, Georgia
}

\begin{abstract}
A serial mental-rotation (MR) task similar to one developed by Bethell-Fox and Shepard (1988) was used to evaluate adult age differences in encoding, rotation, and decision processes. Older adults' response times were longer in each processing stage, and there were small age differences in rotation-stage slopes. Decision times and error rates increased as a function of rotation angle, and were differentially affected by age. The results are consistent with the hypothesis of age-related loss of information from spatial working memory when rotational transformation is required, and suggest that a proportion of age-related slowing in MR slopes found in simultaneous presentation of pairs of figures may reflect age differences in the speed of postrotational decision processes.
\end{abstract}

The mental-rotation (MR) task has frequently been used to study aging and its effects on the speed of spatial information processing. There are several variants of the task, most requiring a subject to discriminate figural stimuli from their mirror images when the stimuli are physically rotated from a standard orientation. In MR tasks requiring paired comparisons of figures, the difference in orientation (or angle of rotation) between the two stimuli is experimentally varied. Response time (RT) has been shown to be a linear function of the angle of rotation required (e.g., Shepard \& Metzler, 1971). Several studies have found that MR intercepts and slopes are greater in older adults than in young adult subjects (e.g., Berg, Hertzog, \& Hunt, 1982; Gaylord \& Marsh, 1975; Jacewicz \& Hartley, 1987). Findings of age differences in MR slopes have generally been interpreted as evidence for slowing in the rate of mental rotation, consistent with the model for MR task processing advocated by Cooper and Shepard (1978). However, a recent study by Hertzog and Yuasa (1988), using two-dimensional figures, found (1) age differences in MR slopes when pairs of stimuli were identical, but not when a stimulus was paired with its mirror image, and (2) large individual differences in the fit of the linear function regressing RT on angle of rotation. Such results suggest that age differences in MR parameters may be complicated by individual differences in MR task strategies, and call into question the assumption that age differences in MR slopes may be attributed to differences in the rate of mental rotation (see Hertzog \& Yuasa, 1988). According to the classic

The present research was supported by a grant to the first author from the National Institute on Aging (R01 AG06123). A version of this paper was presented at the 1990 Annual Meeting of the Psychonomic Society, New Orleans, LA. Address correspondence to the first author, School of Psychology, Georgia Institute of Technology, Atlanta, GA 30332-0170; BITNET: PSCKHCH@GITVM1.
Cooper/Shepard model, slower rate of MR processing should exert equal effects on both same and different responses.

The identification of MR slopes with rotation rate has been questioned in the experimental literature by studies showing that MR slopes are affected by variables such as stimulus complexity (e.g., Folk \& Luce, 1987) and postrotation decision processes (Just \& Carpenter, 1976). To address such issues, Bethell-Fox and Shepard (1988) developed an alternative paradigm designed to separate the MR task into encoding, rotation, and comparison/ decision stages. Subjects press response buttons to control serial presentation of (1) a reference figure, (2) cues indicating extent and direction of rotation, and then (3) a comparison figure which may or may not be the reference figure rotated to the desired orientation. Subjects are instructed to visualize rotation of the figure according to the cues, and then to compare a mental representation of the rotated figure with the comparison figure presented in the third stage. Although Bethell-Fox and Shepard were primarily concerned with stimulus complexity effects, their major finding of relevance to this study was that rotation rate (estimated from RT during the second stage of the task) was a linear function of angle (as in the standard MR task), but that there were no angle effects on encoding or decision times. They also showed that practice with the figures eliminated the complexity effect on rotation-phase $R T$.

The present study used a serial MR task similar to Bethell-Fox and Shepard's (1988) in an attempt to isolate age differences in rotation rate from age differences in decision times. The primary purposes were to determine (1) if age $\times$ rotation angle interactions would be observed in both rotation and decision phases of the task, and (2) if discrepancies between MR slopes for same and different trials would be eliminated in the sequential paradigm. We also hypothesized that age changes in ability to hold a representation of the figure in spatial working memory 
would lead to increasing age differences in MR error rates as a function of increases in the amount of rotation.

\section{METHOD}

The subjects were 19 young adults recruited from Georgia Tech introductory psychology courses and 18 older subjects drawn from the Georgia Tech Cognitive Aging Subject Pool. Older adults were paid $\$ 15$ and students received extra course credit.

\section{Apparatus and Materials \\ Apple IIe microcomputers, equipped with the Digitry CTS firm- ware/software system, were used to present the stimuli on a green monochrome display and record subject reponses. Responses were made on a customized response box containing multiple keys. Figure 1 shows an example of the stimuli. There were three types of comparison stimuli: (1) identical stimuli, (2) mirror image stimuli created by reflection across the vertical axis, and (3) altered (mutant) figures, generated by addi- tions to or omissions from line segments of the standard figure. Mutant figures were used in an attempt to discourage decision making based on incomplete rotations of the standard figures.}

\section{Procedure}

Subjects read instructions from the computer screen, accompanied by the experimenter. They were told that they would be asked to visualize each shape at different angles of orientation by mentally rotating the figure. On each trial, a plus sign (+) first appeared on the screen for 1 sec. Following the warning signal, one of the standard figures appeared on the screen along with an arrow denoting the "top" of the figure (see Figure 2, Panel A). The subjects were instructed to examine every detail of the shape so that they had a "clear, vivid image of the figure" and could "imagine it without actually seeing it on the screen." They were also told to note the position of the arrow. They pressed a response button to indicate readiness to proceed.

Next, the standard shape disappeared from the screen and was replaced by three arrows (Figure 2, Panel B). The first arrow at the very top denoted the direction of rotation (clockwise or counterclockwise). The second arrow at the top was the identical positional cue presented earlier with the standard shape. The last arrow showed where the top of the figure should be imaged after the subject had rotated the figure. Stimuli were to be rotated $0^{\circ}$ (no rotation), $90^{\circ}$, or $180^{\circ}$. Figure 2, Panel B depicts cues for a $90^{\circ}$ (clockwise) rotation.

The subjects pressed a response button after they had mentally rotated the figure according to the arrow cues. The arrows were then replaced by the comparison figure. On same trials, this stimulus was

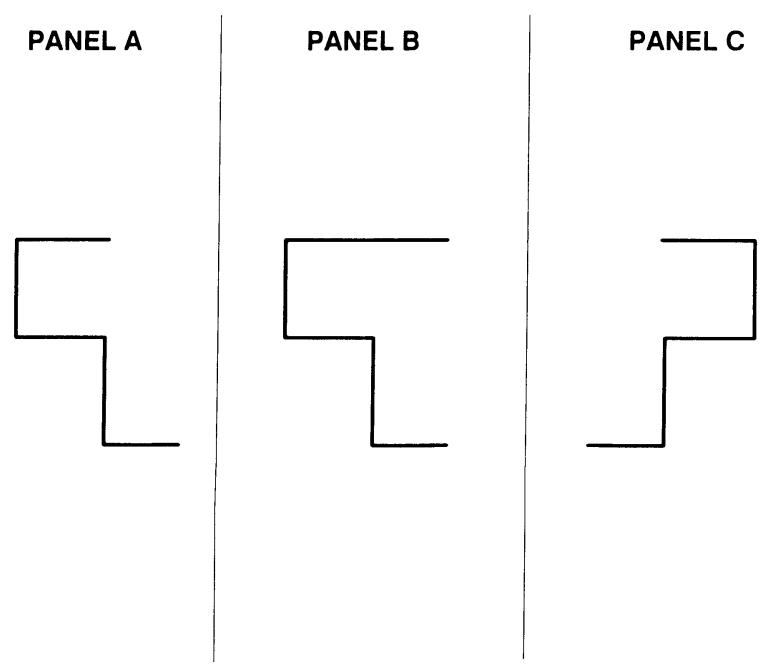

Figure 1. Sample MR stimuli.

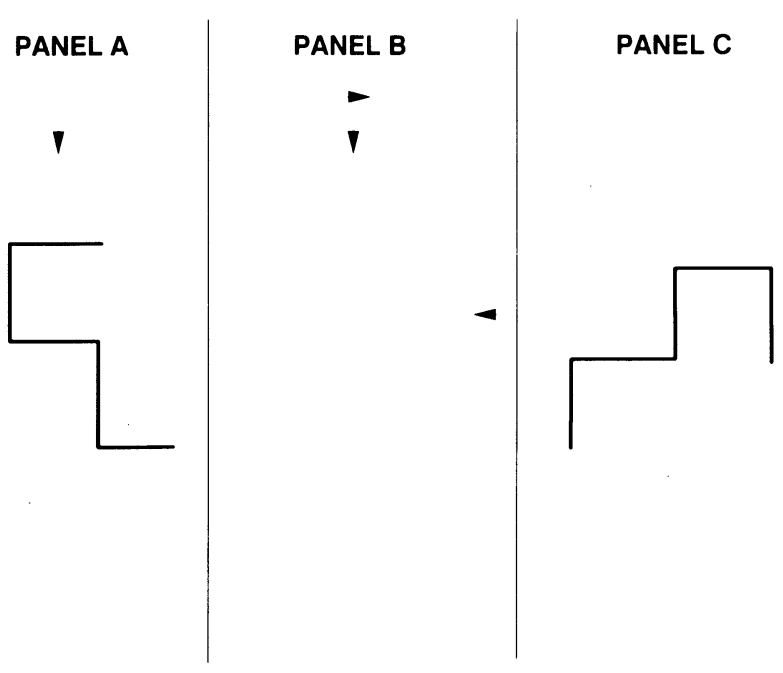

Figure 2. The presentation sequence in the serial MR task: (A) encoding phase, $(B)$ rotation phase, and $(C)$ decision phase.

the standard figure rotated to the orientation indicated by the arrows (shown in Figure 2, Panel C). In this case, the subjects were instructed to press the " $S$ " key. Alternatively, the comparison stimulus on different trials was not the reference figure in the rotated orientation. Instead, the shape was (1) the standard figure rotated to an incorrect orientation (either $90^{\circ}$ more or $90^{\circ}$ less than that indicated by the rotation arrows), (2) a mirror-image stimulus figure rotated to the orientation indicated by the arrow cues, or (3) the "mutant" figure rotated to the orientation indicated by the arrow cues. In each of these cases, the subjects were instructed to press the " $D$ " key. After each decision-phase response, the subjects received accuracy feedback. The instructions placed equal emphasis on speed and accuracy. Each subject received a total of 384 trials.

There were several phases of practice training, giving subjects practice with (1) rotating shapes, (2) rotating shapes and deciding if comparison shapes were at correct or incorrect orientations, and (3) the full task under standard RT conditions (five same and five different trials). On the basis of the results of pilot testing, older subjects were given a more elaborate description of the MR task which aided in their understanding of how to utilize the rotation cues.

\section{RESULTS}

Five older subjects and 1 young subject did not achieve at least $25 \%$ accuracy in all experimental cells (indicating extreme response bias), and hence were eliminated from further analyses. The residual sample included 18 young (10 males, 8 females; age range, $18-22$ years, $M=19.5$ ) and 13 older subjects ( 8 males, 5 females; age range, 54-75 years, $M=63.5$ ). The first set of analyses examined the effects of age and experimental manipulations on individual subjects' median RT for correct responses for each phase of the serial rotation task (encoding, rotation, decision). Data from the three types of different trials were pooled to form a generic same versus different factor.

There were significant age differences in the amount of time spent encoding the standard figure $[F(1,29)=$ $10.64, p<.01$ ], with older subjects taking longer to encode (old $M=2.22 \mathrm{sec}$; young $M=1.08 \mathrm{sec}$ ). Given that the subjects had no information regarding rotation angle during encoding, we did not expect to see any ef- 
fects of angle of rotation in encoding times. However, small linear drops in encoding RT were found $[F(1,29)$ $=7.56, p<.01]$. Subsequent analyses showed that these effects disappeared in the second block of trials, suggesting effects of stimulus learning on early trials to be the source of the effect.

Results from the rotation phase are shown in Figure 3, which shows that RT increased across increasing angles of rotation for both young and older subjects. Trend analysis detected a linear effect across rotation angles $[F(1,29)$ $=66.33, p<.001]$, but, despite the curvature suggested in Figure 3, the quadratic trend failed to reach significance $(p>.05)$. The age $\times$ angle (linear) interaction approached, but did not achieve, significance $[F(1,29)=$ $3.07, p<.10]$, although there was a significant main effect for age. However, the age $\times$ angle (linear) interaction was significant in the second block of trials $(p<.05)$, suggesting that variability in early performance increased experimental error and obscured the effect. Older adults took more time in the rotation phase and somewhat more time to rotate the mental image of the reference figures. No other main effects or interactions were significant.

Figure 4 plots the decision-phase RTs across angle of rotation for each age group. As in the rotation phase, RTs in the decision phase increased with increasing angles of orientation, with significant linear and quadratic trend components. There was a significant main effect for age, with older subjects taking longer to decide than young subjects. The increase in decision RT with rotation angle was greater for the older than for the young subjects, as indicated by a significant age $X$ angle (linear) interaction $[F(1,29)=7.08, p<.01]$. Same trials produced shorter RTs than different trials $[F(1,29)=4.99, p<.05]$, but no other interactions were significant.

\section{Accuracy Data}

The patterns of the proportion of correct decisions in the decision stage are shown in Figure 5. Accuracy decreased linearly as a function of rotation angle $[F(1,29)$

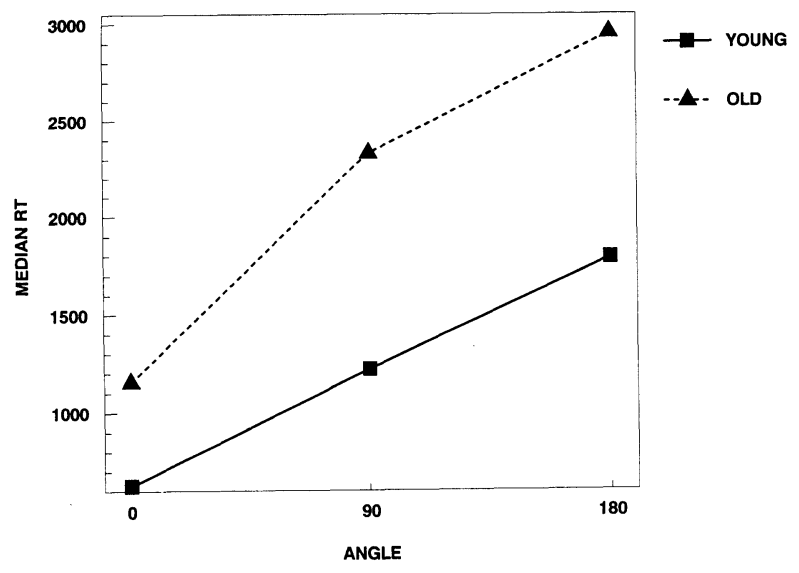

Figure 3. Age differences in mean rotation-phase RT across rotation angles.

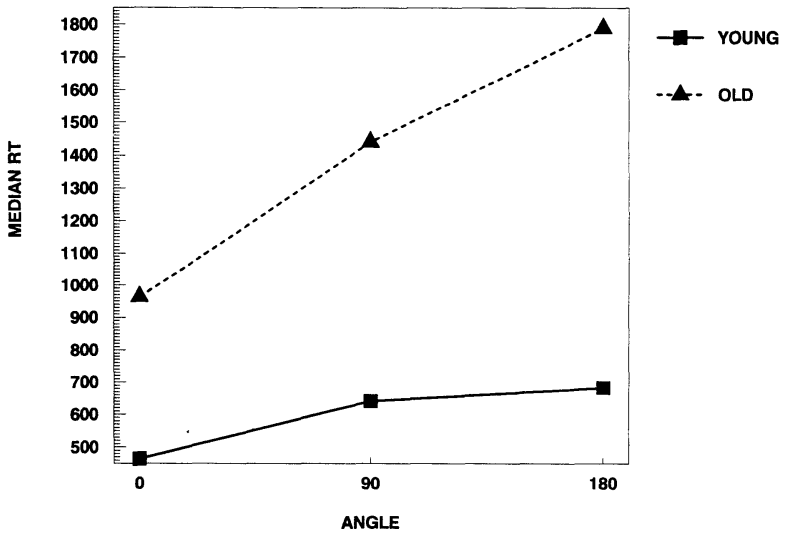

Figure 4. Age differences in mean decision-phase RT across rotation angles.

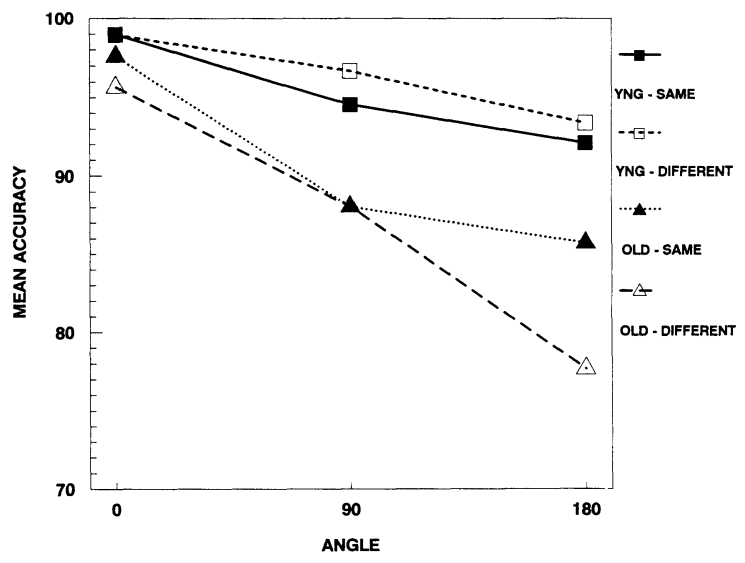

Figure 5. Age differences in proportion correct for same and different trials across rotation angles.

$=101.37, p<.001]$, with greater decreases for different than for same trials $[F(1,29)=4.55, p<.05]$. Although the overall quadratic trend for angle was not statistically reliable, the quadratic angle $X$ sameffidifferent interaction was significant $[F(1,29)=7.27, p<.01]$, reflecting a trend for greater curvilinearity in proportion correct for different trials. On average, older subjects made more errors than young subjects $[F(1,29)=8.83$, $p<.01]$, and the significant age $\times$ angle (linear) interaction indicated that older adults' accuracy decreased more across increasing angles of rotation than did the accuracy of young adults. The pattern of results was further complicated by a significant age $\times$ angle (linear) $\times$ same $/$ different interaction $[F(1,29)=10.63, p<.01]$, indicating that age differences in magnitude of declines in accuracy with increasing rotation angle were larger for different trials than for same trials.

\section{DISCUSSION}

The results of this experiment, in which an alternative paradigm was used to study mental rotation, are intriguing when compared with the 
results of studies in which paired comparisons of rotated geometric figures were used. Berg et al. (1982) found substantial age differences in the MR RT slopes for same trials, as did Hertzog and Yuasa (1988). The latter found, however, that age differences in different slopes were not statistically significant unless subjects with poor-to-moderate fits of the linear function to different trials were eliminated from the analysis (see also Gaylord \& Marsh, 1975). In the usual paired comparison task, the rate of rotation is presumed to be reflected in the MR slopes, but can be influenced by differences in nonrotational processes that vary across angle of rotation. The serial MR task yielded no differences between same and different trials in either the rotation phase or the decision phase in the pattern of RT increases across angles of rotation. There were age $X$ angle interactions in both rotation- and decision-phase RTs, as well as in decision-phase error rates. The latter result is similar to the findings of Clarkson-Smith and Halpern (1983).

The most plausible interpretation of this pattern of results is an agerelated degradation in the quality of the mental representation of the reference figure, occurring either during the MR phase or after presentation of the comparison figure at the onset of the decision phase of the trial. The longer the rotation to be visualized, the greater the likelihood of degraded quality of stimulus representation, or perhaps even complete loss of figural information in spatial working memory, for older persons. Aging is known to affect working memory (Salthouse, 1988).

Degraded representation of the figure could also account for the age differences in decision-phase RT. It is well known that decision time varies inversely with both quality or strength of the stimulus and the confidence level of the subject (Luce, 1986). These results appear to conflict with Bethell-Fox and Shepard's (1988) finding of no effects of rotation angle on decision-phase accuracy or RT. The differences in findings regarding decision-phase RT may be due to the fact that BethellFox and Shepard imposed a response deadline on subjects in the decision phase of their experiment. That procedure was not employed in the present study, given difficulties inherent in selecting different response deadlines for the two age groups, as well as our desire to maintain comparability of decision-phase MR RT in this paradigm with the standard MR RT task.

The comparison is actually intriguing. Given no age differences in the pattern of same and different RTs in the serial MR task, in contrast to Hertzog and Yuasa's (1988) results with the standard simultaneous MR task, we hypothesize that a substantial proportion of age differences in MR RT is associated with a postrotation decision-verification stage, in which subjects continue to compare congruent stimulus features prior to responding (Just \& Carpenter, 1976). The standard MR task, with its simultaneous presentation of the two figures (which remain physically present until the subject responds), affords this type of verification strategy. The serial task discourages additional decision-verification processing because one can engage in further processing of the rotated representation of the reference figure only in spatial working memory, which may be susceptible to replacement or decay. The interaction with type of trial (same vs. different) could be explained by a processing model that incorporates different criteria for each response type (see, e.g., Ratcliff, 1985). A diffusion model could account for these differences by hypothesizing that (1) subjects have a more conservative response criterion for responding "different" than for responding "same," (2) the time needed for comparison of feature representations increases as a function of rotation, and (3) the criteria differ across levels of chronological age. Other models, including ones positing qualititative differences in processing strategies for same and different trials across different individuals, could also account for the results. Clearly, further research with versions of both kinds of MR tasks would be needed to address these issues. Nevertheless, this study suggests the possibility that previous inferences regarding age differences in the rate of mental rotation may have been based on empirical findings that were determined in part by age differences in the rate of nonrotational processes.

\section{REFERENCES}

Berg, C., Hertzog, C., \& Hunt, E. (1982). Age differences in the speed of mental rotation. Developmental Psychology, 18, 95-107.

Bethell-Fox, C. E., \& ShePard, R. N. (1988). Mental rotation: Effects of stimulus complexity and familiarity. Journal of Experimental Psychology: Human Perception \& Performance, 14, 12-23.

Clarkson-Smith, L., \& Halpern, D. F. (1983). Can age-related deficits in spatial memory be attenuated through the use of verbal coding? Experimental Aging Research, 9, 179-184.

COOPER, L. A., \& SHEPARD, R. N. (1978). Transformations on representation of objects in space. In E. C. Carterette \& M. Friedman (Eds.), Handbook of perception (Vol. VIII): Perceptual coding (pp. 105-146). New York: Academic Press.

FolK, M. D., \& LUCE, R. D. (1987). Effects of stimulus complexity on mental rotation rate of polygons. Journal of Experimental Psychology: Human Perception \& Performance, 13, 395-404.

GAYLORD, S. A., \& MARSH, G. R. (1975). Age differences in the speed of a spatial cognitive process. Journal of Gerontology, 30, 674-678.

HerTZOG, C., \& YUASA, M. (1988). Adult age differences in speed and accuracy of mental rotation. Unpublished Manuscript.

JACEWICZ, M. M., \& HARTLEY, A. A. (1987). Age differences in the speed of cognitive operations: Resolution of inconsistent findings. Journal of Gerontology, 42, 86-88.

Just, M. A., \& CARPENTER, P. A. (1976). Eye fixations and cognitive processes. Cognitive Psychology, 8, 441-480.

LUCE, R. D. (1986). Response times: Their role in inferring elementary mental organization. New York: Oxford University Press.

RATCLIFF, R. (1985). Theoretical interpretations of the speed and accuracy of positive and negative responses. Psychological Review, 92 , 212-225.

Salthouse, T. A. (1988). The role of processing resources in cognitive aging. In M. L. Howe \& C. J. Brainerd (Eds.), Cognitive development in adulthood (pp. 185-239). New York: Springer.

ShePARD, R. N., \& METZleR, J. (1971). Mental rotation of three dimensional objects. Science, 171, 701-703.

(Manuscript received for publication October 27, 1990.) 